\title{
Olmesartan-induced enteropathy resembling celiac
} disease

A 70-year-old woman with longstanding lymphocytic colitis presented at an outside hospital with worsening diarrhea (up to 30 bowel movements per day), epigastric pain, and $30 \mathrm{lb}(13.5 \mathrm{~kg})$ weight loss which had required multiple admissions since July 2012. Multiple courses of oral steroids were tried with no relief. Because of severe villous atrophy and mucosal scalloping found on esophagogastroscopy, she was diagnosed with "celiac disease" and was placed on a gluten-free diet, but without much improvement.

Celiac serology tests were negative. Human leukocyte antigen testing was negative. Computed tomography (CT) scan showed diffuse wall edema and thickening of the jejunum and ileum ( $\bullet$ Fig. 1 ). The colon was endoscopically normal. Push enteroscopy revealed severe villous atrophy with visible submucosal vessels in the jejunum and severe scalloping and atrophy in the duodenum ( $\bullet$ Fig. 2). Capsule endoscopy showed severe ileojejunitis with mucosal ulceration and villous atrophy ( $\bullet$ Fig. 3; $\bullet$ Video 1, $\bullet$ Video 2 ). Biopsies revealed severe villous atrophy with no significant increase in intraepithelial lymphocytes. The patient denied taking olmesartan, but when the brand name was mentioned she agreed that she had used it for 2 years. Her symptoms improved after the medications were stopped.

Olmesartan (an angiotensin receptor blocker often used to treat hypertension) has recently been associated with a sprue-like enteropathy [1]. The Food and Drug Administration has only recently issued a warning regarding this condition. As more cases are identified, the exact mechanisms may become clear, although a cell-mediated immune response is thought likely [1]. Our case is the first panendoscopic characterization of olmesartan-induced enteropathy presented so far, demonstrating the villous atrophy on push enteroscopy and capsule endoscopy. Awareness of this condition is critical to identifying the inciting factor and allowing healing in cases that may otherwise be missed.

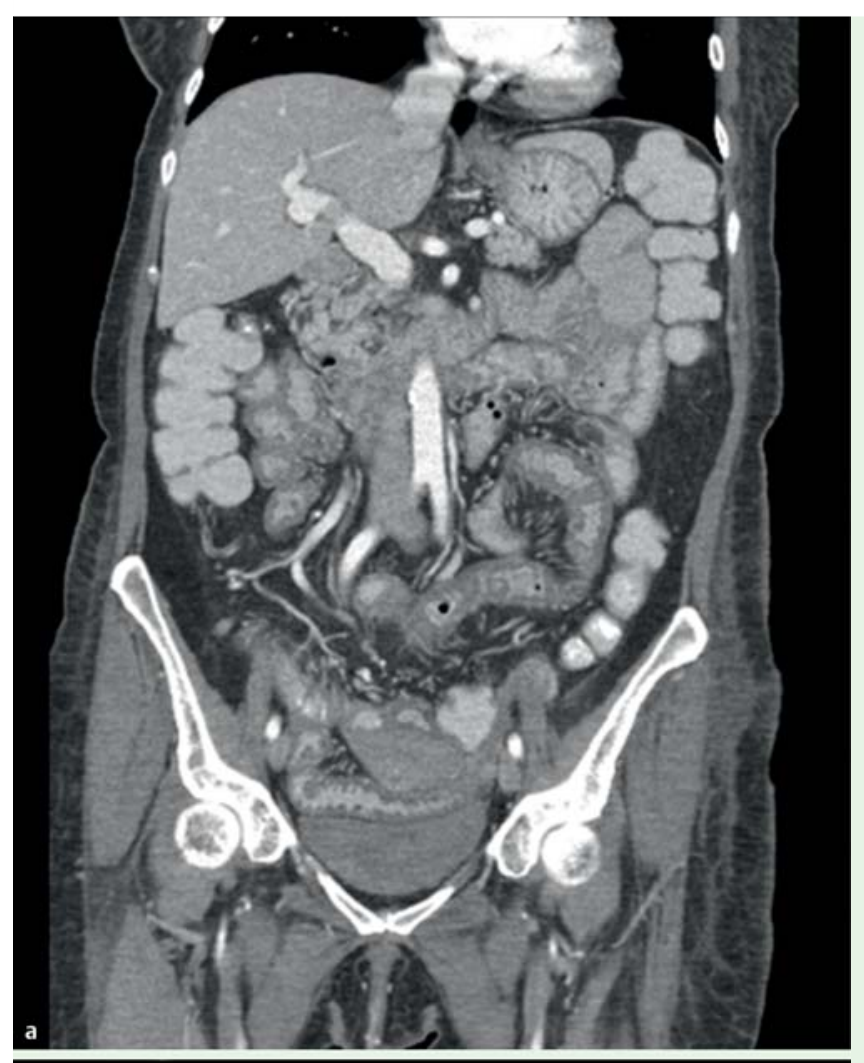

Fig. 1 Computed tomography $(\mathrm{CT})$ of abdomen and pelvis showing severe diffuse bowel wall edema and thickening of the jejunum and ileum in a 70-year-old woman with longstanding lymphocytic colitis.

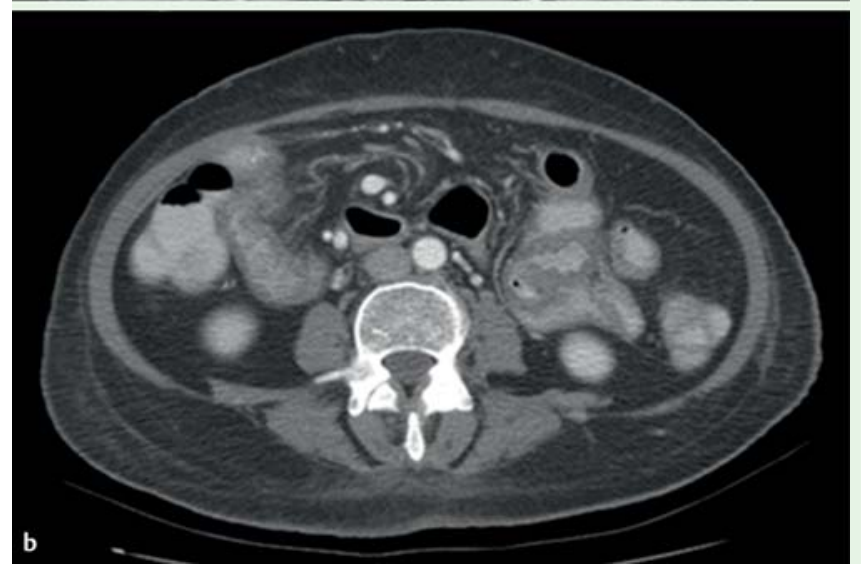

Video 2

\section{Video 1}

Capsule endoscopy demonstrating severe mucosal ulceration and villous atrophy in the jejunum of a 70-year-old woman with longstanding lymphocytic colitis.
Capsule endoscopy demonstrating severe mucosal ulceration and villous atrophy in the ileum of a 70-year-old woman with longstanding lymphocytic colitis. 


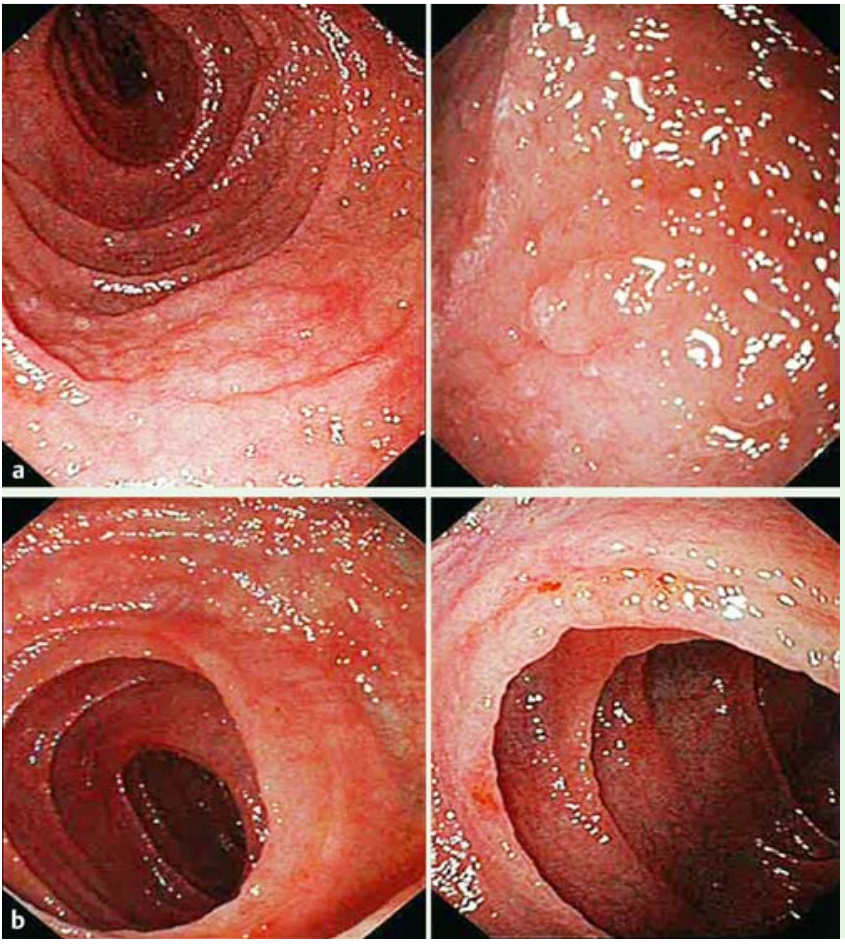

Fig. 2 a, b Push enteroscopy showing a severe villous atrophy with visible submucosal vessels in the jejunum and $\mathbf{b}$ severe scalloping and atrophy in the duodenum.
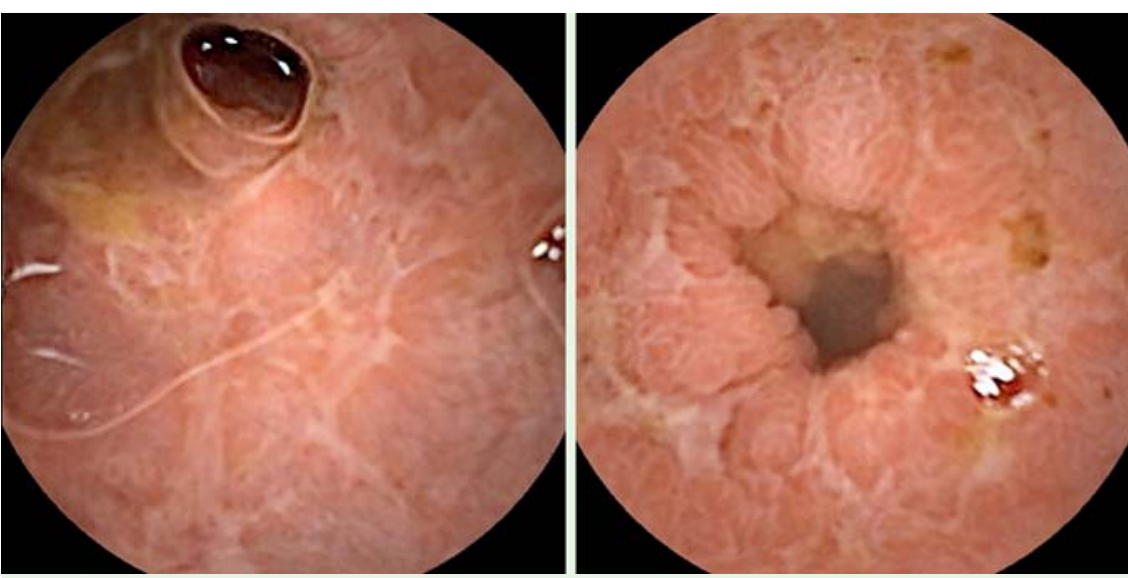

Fig. 3 Capsule endoscopy demonstrating severe ileojejunitis with mucosal ulceration and villous atrophy.
Endoscopy_UCTN_Code_CCL_1AC_2AH

\section{Competing interests: None}

\section{Ali Safdar Khan, Shajan Peter, C. Mel Wilcox}

Division of Gastroenterology and Hepatology, University of Alabama at Birmingham

\section{References}

1 Rubio-Tapia A, Herman ML, Lu JF et al. Spruelike enteropathy associated with olmesartan. Mayo Clinic Proc 2012; 87: 732 - 738

\section{Bibliography}

DOI http://dx.doi.org/

10.1055/s-0033-1359132

Endoscopy 2014; 46: E97-E98

(c) Georg Thieme Verlag KG

Stuttgart · New York

ISSN 0013-726X

\section{Corresponding author} Ali Safdar Khan

University of Alabama at Birmingham Gastroenterology and Hepatology

1720 2nd Ave South BDB 372

Birmingham 35294

United States

Fax: +1-205-975-6381

ali.safdar@gmail.com 